In Crescendo, 2019; 10(4): 653-666

Fecha de recepción: 25/05/2019

Fecha de aceptación: 15/12/2019

\title{
EL APORTE DEL EMPRENDIMIENTO SOCIAL A LA EDUCACIÓN MEDIA TÉCNICA
}

\author{
THE CONTRIBUTION OF ENTREPRENEURSHIP TO \\ SECONDARY TECHNICAL EDUCATION
}

Luisa Fernanda Cadena-Corredor ${ }^{1}$

\section{RESUMEN}

El presente artículo recoge algunos de los principales resultados generados de la investigación ejecutada en el periodo 2014-2017 en 4 instituciones de educación media técnica del sector oficial en la ciudad de Bogotá, en la que se pretendió comprender el impacto de las políticas de articulación de la media t y la formación en emprendimiento que desde el Ministerio de Educación Nacional hacen parte del Proyecto Educativo Institucional (PEI) de las instituciones que tienen en su modelo educativo la formación media técnica.

Esta indagación se conduce bajo la mirada del desarrollo del pensamiento complejo en la escuela y con ella se quiso conocer cómo impactaba en el desempeño de los egresados de la formación media en articulación y la implementación de las dos políticas públicas que se plantean para mejorar la formación del futuro egresado como bachiller técnico para el ingreso al sector productivo o bien para su paso a la formación superior.

En cuanto a la metodología desarrollada en el estudio, la investigación fue de tipo cualitativo, con enfoque descriptivo.

Para organizar y analizar la información obtenida se realizó el proceso metodológico de triangulación, con el objetivo de darle la rigurosidad científica a los resultados de la investigación recogidos desde los diferentes actores, mediante el análisis de los resultados arrojados.

1 Docente de posgrado Universidad Santo Tomas, Docente de posgrado CEMIL Centro de Educación Militar, Docente de Educación media técnica SED Bogotá. 
En conclusión, la investigación entregó entre otros aportes una serie de componentes para generar un proyecto de aula a partir del desarrollo de competencias en emprendimiento social. PALABRAS CLAVE: Educación media, política de articulación, sector productivo, pensamiento complejo, competencias.

\section{ABSTRACT}

This article presents some of the main results obtained from research developed in four institutions of secondary technical education of the public sector in Bogota, during 2014 2017. The research aimed to understand the impact of policies related to the implementation of the secondary education and training in entrepreneurship, introduced by the Ministry of Education, which are part of the PEI (institutional educational project) institutes that have in their educational model the secondary technical formation.

This inquiry was conducted under the view of the development of complex thinking in schools, and through it, it was wanted to know how the implementation of public policies, which were established to improve the formation of the future technical high school graduated towards either a future introduction to the labor force or to continuing to pursue higher education, impacted in the performance of the graduated students of the secondary formation in articulation.

In regards to the methodology developed in this study, this was a qualitative research, with a descriptive approach.

In order to organize and analyze the information, a triangulation process was performed. This, with the aim to achieve scientific thoroughness in the final research results, collected from the different participants, through analysis of the obtained results.

In conclusion, among other contributions, this research provided a series of components to generate a class room project, from the development of competencies in social entrepreneurship.

KEY WORDS: Secondary education, articulation policy, productive sector, complex thinking, competencies.

\section{INTRODUCCIÓN}

La política de articulación de la educación media-técnica con la educación superior, es llevada a cabo en Colombia desde el año 2001 hasta la fecha y tiene como propósito profundizar la formación en competencias laborales y formación en emprendimiento de los jóvenes que estén próximos a terminar el ciclo de educación secundaria y pueden pasar a formar parte de la oferta laboral con la que cuenta el sector productivo o continuar su formación en la educación superior. 
Por otra parte, la política de fomento a la cultura del emprendimiento, formulada en la Ley 1014 del 2006 ordena a las instituciones educativas del país, oficiales y privadas trabajar de manera transversal la cultura del emprendimiento en todos los niveles de educación, para brindar formación integral en valores como desarrollo del ser humano y su comunidad, autonomía, sentido de pertenencia, trabajo en equipo, desarrollo de la innovación y deseo por el aprendizaje permanente.

Sin embargo, después de más de 10 años que los colegios oficiales de Bogotá hacen parte del proceso de articulación de la educación media con el Servicio Nacional de Aprendizaje (SENA), e instituciones de educación superior (IES), los resultados arrojados en la aplicación de las pruebas Saber 11, así como el seguimiento realizado al desempeño laboral y académico de los egresados, suponen la baja calidad de la preparación de los estudiantes impartida en estas instituciones. De estos resultados no escapa el proceso de formación media en articulación de las instituciones indagadas, aun cuando desde los entes gubernamentales se promueve como ejemplo de calidad educativa cerrando brechas en acceso a la educación superior.

El ingreso a la formación superior en el territorio colombiano continúa siendo un privilegio de pocos debido a que los cupos en entidades oficiales no son suficientes y los bachilleres egresados del proceso de articulación en su gran mayoría no pueden asumir los costos de una educación privada. Por otro lado, la oferta laboral no brinda suficientes puestos de trabajo para las modalidades de formación técnica en la que se especializaron durante el programa de articulación.

Bajo la legislación colombiana se consideran jóvenes las personas que se encuentran entre los 14 y 28 años de edad según la Ley 1622 de 2013, o Ley del "estatuto de ciudadanía juvenil".

Revisando las proyecciones de tasa poblacional presentada por el Departamento Administrativo Nacional de Estadística (DANE), la población juvenil del país aumenta de manera constante de manera que para este 2018 el porcentaje de jóvenes es de 27,4\%, representado en 13,735,084 jóvenes preparados para trabajar o continuar su formación superior; de estos, más del 41,5\% (5,700.000) de jóvenes se encuentran inactivos de acuerdo con el reporte del programa de la presidencia Colombia joven (2017) y estadísticas presentadas por el Departamento Nacional de Planeación (DNP), en abril del presente año. (DNP D. N., 2016). 
Según el DNP, la desocupación del país está representada en la mitad de la estadística general por las personas menores de 29 años que no logran acceder a un empleo o no consiguen que hacer, es decir que, de cada dos colombianos sin empleo, uno es menor de 29 años.

Anualmente se gradúan en el país cerca de 500.000 bachilleres cada año, de los cuales un porcentaje inferior al $30 \%$ continúa de manera inmediata en la educación superior y de acuerdo con el MEN (2016) de este porcentaje apenas un $20 \%$ ingresa a la educación profesional y el otro 10\% tiene como opción el ingreso a la formación técnica y tecnológica; según los datos del Sistema Nacional de Información de la Educación Superior (MEN S. , S.F.)

Frente a la situación enunciada, es relevante pensar el proceso de educación media de manera diferente. Esta debería ser un proceso de educación personalizada donde se detecten las dificultades y fortalezas individuales de los estudiantes y se promuevan procesos dinámicos, activos e interactivos, en un modelo de educación encadenada, cumpliendo los contenidos contemplados pero de la mano de un desarrollo personal que lleve a enfrentar incertidumbres, a tomar riesgos, a tomar decisiones y a hacer elecciones, es decir formar en la literacidad profesional necesaria para vivir en sociedad, lo cual genera un nuevo compromiso vital de la educación.

Paralelamente es consecuente reflexionar sobre el papel de la política de articulación que se planea desde el modelo de educación estatal en la separación y reduccionismo de los contenidos, para gestionar en cambio una propuesta de formación considerada como la oportunidad para forjar un proyecto de vida digno a través de la consecución de saberes compartidos que garanticen una buena convivencia y una vida feliz para los educandos, pues como lo menciona el pensamiento complejo enseñar a vivir "es introducir una cultura de base que comparte el conocimiento del conocimiento"(Morin, Edgar, 2014, pág. 16)

El escenario de la educación demanda al docente a generar momentos académicos, ayudándose de técnicas, recursos y espacios, para articular la teoría con las experiencias, partiendo siempre de los intereses y necesidades particulares de los estudiantes que promuevan en ellos la necesidad de generar su propio conocimiento.

En su rol orientador, el docente está en el deber de estimular a los estudiantes a generar un pensamiento de tipo universal, práctico y complejo, lo cual es fundamental en la formación de ciudadanos líderes sociales, interesados en los problemas de su entorno y deseosos de generar soluciones desde un ambiente 
académico, que favorezcan el desarrollo individual, social, económico, político, cultural y ecológico, implementando así una educación comprometida en desarrollar las dimensiones y manifestaciones humanas. (Morin, Edgar, 2014).

Por otro lado, la escuela actual es parte de un modelo social que vive en continuo movimiento, en donde nada es perdurable, puesto que existe la nueva modernidad líquida la cual permea cada espacio, lugar, momento y condición de docentes y estudiantes. Este modelo de modernidad está llevando a cada persona a circular por diferentes maneras para formalizar las relaciones sociales y afectivas, indistintas fuentes de trabajo con un alto grado de desarrollo competitivo, inmersas en un modelo de vida individualista, con una mentalidad fútil en valores.

Quizás una de las consecuencias más fuertes de esta modernidad liquida, según Bauman, reside en evadir el compromiso para adquirir responsabilidad por los actos realizados, puesto que "la instantaneidad lleva a la ética humana a un territorio inexplorado, donde la mayoría de los hábitos aprendidos para enfrentar la vida han perdido toda utilidad y sentido" (Bauman, Zygmunt, 2007, pág. 137).

Esta misma instantaneidad acostumbra a los protagonistas a una vida de aprendizaje rápido donde olvidar y aprender es igual de importante cuando la persona trata de encontrarse a sí misma en un mundo en el cual el largo plazo no se construye. La nueva generación liquida convive además con otro componente de la modernidad que es el consumo y ser un ente consumidor acarrea también procesos de aprendizaje; aunque aprendizaje rápido, requiere de habilidades y destrezas aprendidas, desaprendidas y reaprendidas con la velocidad del renuevo de la información suministrada por los medios masivos.

Entonces, ¿Dónde queda el tiempo necesitado para reflexionar sobre las diferentes enseñanzas? Por esto mismo se asume que será un acto complejo el campo de la educación y por ende el tratar de generar un cambio de pensamiento en las nuevas generaciones.

Consecuentes con el momento de instantaneidad que vivimos todos, es de considerar que el proceso de formación de bachilleres se ajuste a las exigencias actuales de un mundo globalizado y es en este contexto que debe pensarse en brindar una formación holística, integradora y compleja, que supere la mera preparación técnica en un desempeño específico. El emprendimiento social aporta innumerables elementos en la construcción del conocimiento y en el proceso de reaprender a la velocidad de los cambios modernos "porque debemos aspirar al menos, a un conocimiento multidimensional” (Morin, Edgar, 2011, pág. 152). 


\section{Aporte del Emprendimiento social}

Frente a la desarticulación de saberes identificada en la formación mediasuperior y a pesar de la incursión de la política de articulación en esta, se propone responder desde el planteamiento de la UNESCO aprender a aprender y aprender a vivir juntos, o, desde el pensamiento de Morín articular los saberes de manera recursiva aprendiendo-desaprendiendo-reaprendiendo. Es decir, dándose la posibilidad de hacer de este ciclo de educación algo diferente, gracias a que es el estudiante quien se pregunta y cuestiona por su formación y no espera que sea otro el que decida el camino que debe recorrer.

Para ayudar a realizar este paso se toma el concepto de currículo complejo de Gonfiantini, como un modelo de construcción compleja e intersubjetiva del conocimiento, "donde el mismo no se transmite sino que es construido deconstruidoreconstruido en un permanente diálogo recursivo mediante la acción de los distintos actores" (Gonfiantini, 2016) por lo cual se conceptualiza en la implementación de la política para la formación en competencias emprendedoras de los estudiantes en la educación media técnica.

¿Cómo se plantea el emprendimiento en educación? En un entorno complejo colmado de presunciones e imperfecciones políticas las memorias históricas nos traen desde la propuesta de construcción de conceptos de Sócrates, que aún sustenta parte del quehacer educativo, hasta la educación fundada en competencias instalada en los países que se familiarizan con teorías prestadas que aspiran dar alcance a las propuestas del conocimiento.

Por lo tanto, trasladar el concepto de emprendimiento social al entorno educativo es una apuesta a romper el statu quo de procesos que desde hace décadas desvertebran los procesos de concienciación que son básicos en la educación de espíritus libres y líderes. (Cadena, 2017).

Heidegger, M. en su lucido estudio denominado "habitar para construir, construir para habitar", elabora una propuesta con la cual viene a colaborar y afianzar de manera epistemológica este diseño. Asegura que la historia del desarrollo de la humanidad esta solapada en un contrasentido que sustenta el planteamiento propuesto a continuación: Generar una formación del emprendimiento social con la cual se fortalezcan herramientas que conduzcan a habitar un mundo en constante transformación. 
Más allá de simplemente generar una propuesta más, se espera establecer un punto de discusión en torno a la calidad académica de millones de estudiantes y futuros bachilleres frente al futuro que por cuenta de los desarrollos tecnológicos ven un panorama oscuro en la luz que debería ser la educación. Por ello, las nuevas pedagogías deben visibilizar los retos a los cuales se enfrenta el estudiante del presente y a partir de allí construir un futuro con ciertos visos de claridad que aporte a la conformación de una sociedad sostenible y más humana.

Generar una propuesta de formación que contemple los principios del emprendimiento social implica contemplar diversos intereses, preocupaciones, necesidades y deseos tanto de orden institucional, como académico y profesional. Para obtenerlo, se ha partido del carácter de las instituciones educativas indagadas previamente en esta pesquisa, así como del sentir recogido de estudiantes, de los egresados de la formación media técnica en articulación, del grupo de docentes, directivos y padres de familia; frente a los elementos que consideran debe contener un modelo de educación que brinde una formación interesada en terminar con el reduccionismo y tecnicidad implantados y en cambio promueva una formación esencial, humanista y científica con visión global del mundo moderno en el cual los educadores sean protagonistas fundamentales del cambio educativo. (Morin, Edgar, 2011).

\section{El emprendimiento y el emprendedor}

El emprendimiento es el campo donde se lleva a cabo la búsqueda y la comprensión de los comportamientos y actitudes de personas que reúnen las características necesarias para impulsar proyectos que generan organizaciones cuyo proceso de desarrollo permite llevarlas a su crecimiento. (Cadena, 2017)

Emprendedor es la persona que prueba poseer la habilidad para identificar las oportunidades de mejoramiento y transformación, así como la viabilidad para lograr la movilidad de recursos necesarios en la ejecución de una oportunidad detectada. Generalmente se describe a un emprendedor como el individuo dinámico, inquieto, arriesgado, creativo y con deseos de crecimiento. En consecuencia, es un individuo que sabe organizar las relaciones necesarias entre personas y recursos para alcanzar un fin personal o colectivo o para tener el control de una situación (Cadena, 2017) porque constantemente identifica diferentes situaciones que pueden ser generadoras de oportunidad de desarrollo, avance y progreso personal siendo además generador de valor social. 


\section{Emprendimiento social}

El emprendimiento social ha llamado notablemente la atención en el mundo académico, razón por la cual es fácil encontrar numerosas líneas de investigación dentro de este campo. Ha sido tratado por algunos autores como Dees, como una nueva manera de producir conocimiento enfocado en disminuir la pobreza mundial y plantear soluciones a problemas sociales como los cambios sufridos en la población, la poca eficiencia de los organismos gubernamentales, la emancipación en la forma de desarrollo en un mundo globalizado y la falta de satisfacción de las necesidades de los diferentes sectores sociales, los cuales son detonantes para el impulso de los proyectos de emprendedores sociales. (Dees, 2001).

El emprendimiento social puede entenderse como la especialidad que se encamina a la formulación e implementación de propuestas sostenibles que plantean respuestas efectivas a dificultades identificadas en un grupo social para lograr un beneficio común.

Entonces, si el concientizar en la responsabilidad social es el objeto de interés del emprendimiento social, ¿Cuál es el papel que desempeñan las instituciones de educación media? Puesto que son las interesadas en impulsar un modelo de formación contemporánea en donde no se fragmenten los saberes, sino que se trabaje por generar un pensamiento complejo dado que "solo un pensamiento complejo puede darnos armas para preparar a la metamorfosis social, individual y antropológica. (Morin, Edgar, 2011, pág. 143).

Se presume que las instituciones atiendan desde sus programas las necesidades específicas de aprendizaje para la formación de jóvenes bachilleres sin anular la curiosidad de estos, garantizando los saberes pertinentes, asumiendo incertidumbres y recobrando la ética en la raza humana.

En los muchos estudios realizados por Kliksberg, acerca de la pobreza y el desempleo juvenil en América Latina, relata frívolamente la situación que actualmente viven los cientos de jóvenes latinoamericanos entre los 13 y 19 años, que son excluidos del sistema educativo y del mercado laboral. "Se los llama jóvenes marginales, pero el nombre correcto es jóvenes excluidos: la sociedad no les hace lugar." (Kliksberg, 2011).

Cuando los jóvenes no pueden conseguir un trabajo digno ni tampoco pueden lograr formar un grupo de relaciones sociales, ni integrarse a un grupo socialmente conformado, se genera esta exclusión. Es así que la falta de preparación, la 
falta de educación, la baja o nula instrucción en un oficio específico, inhibe para cualquier persona el acceso a un empleo, el acceso a un trabajo digno y el acceso a las mínimas condiciones dignas de vida. Dice Kliksberg, que los jóvenes se sienten «acorralados» en una sociedad que de manera injusta les limita el acceso a emplearse y ser socialmente productivos.

El desempleo juvenil mantiene características preocupantes en Colombia, entre las cuales se destacan el desajuste entre los programas de educación y la oferta laboral, así como el alto porcentaje de los NINI (jóvenes que ni estudian, ni trabajan, ni se preparan) entre los jóvenes. En cuanto a la primera característica se analiza que no existe la relación directa entre mayor preparación y mayor acceso a un empleo acorde o calificado. En cuanto a la participación de los NINI, en la tasa de desempleo, la OIT refiere que uno (1) de cada cinco (5) jóvenes, no está trabajando ni estudiando, ni recibiendo formación técnica y está colaborando en labores domésticas. (MEN M. d., 2016). La insuficiencia en la atención de educación y la carencia de una formación laboral complica a los NINI en la obtención de un empleo en el futuro, relegándolos para acceder al mercado laboral y además a los grupos sociales.

De acuerdo con las definiciones de Kliksberg sobre la responsabilidad social y el papel del ciudadano común, se concluye que lograr la transformación social es responsabilidad de todos y es por esto que la sociedad debe crear las condiciones necesarias para que la familia tenga derecho a existir como tal. La pobreza, la falta de oportunidades laborales y la negación de acceso a la educación especializada, acarrea desarticulación de la familia.

La escuela debe intensificar su misión de formar jóvenes y ciudadanos en valores. Debe insistir en la formación desde el respeto, en el amor propio y la aceptación de las diferencias, como también enfocar sus esfuerzos en formar personas con capacidad de perdón y de reconciliación, con un alto deseo de generar ideas de desarrollo y crecimiento personal y comunitario.

Este objetivo de generar valor social se sustenta en el conjunto de habilidades y destrezas que poseen las personas cuya formación implique formar para la literacidad profesional apoyados en el emprendimiento social, y que pueden ser entre otras:

- Resiliencia, pues el emprendedor social debe estar preparado para afrontar frustraciones personales, familiares e incluso sociales y ser capaz de sobreponerse a estas para continuar en el desarrollo de su misión. 
- Debe poseer innovación, pues constantemente debe generar ideas novedosas para dar soluciones a situaciones que en ocasiones son recurrentes.

- Responsabilidad social, en cuanto su objetivo será siempre generar una respuesta positiva que genere bienestar personal y comunitario.

- Compromiso: el emprendedor debe estar siempre atento, perseverando en la consecución del objetivo, animando a los otros, obviando obstáculos, trabajando para alcanzar lo planteado.

- Liderazgo: debe poseer la capacidad de dirigir y guiar a un equipo tras la consecución de un objetivo de bienestar común.

- Autoconfianza: conocerse suficientemente para tener confianza en sí mismo y conocer el alcance de sus posibilidades.

- Capacidad de asumir riesgos, en tanto emprender un proyecto siempre tendrá un alto nivel de incertidumbre, se puede pasar por momentos de duda y temor, pero se debe estar preparado para asumir los contratiempos y reponerse a estos para continuar con la convicción que se puede lograr el cumplimiento de las metas.

Estos componentes sustentan la matriz de la propuesta para aportar a una educación media técnica en articulación a la superior que genere una personalidad emprendedora capaz de desafiar la realidad al someter a prueba las capacidades para resolver un problema social, que puede no ser relevante para muchos, o sencillamente no ser identificado o dimensionado por la comunidad al ser parte de este.

Porque aprender no es solo adquirir; es el saber hacer adquisición del saber (...). El aprendizaje es un proceso evolutivo espiral, regido por la dialógica autoeco-organizadora, y en la que los términos de innato/adquirido/construido se encadenan, permutan y producen entre sí. El desarrollo del saber va de la mano con el desarrollo de las aptitudes para adquirir, memorizar y tratar el conocimiento (Morin, Edgar, 2011) De esta afirmación se entiende que aprender implica desarrollar la unión de lo conocido y lo desconocido a través del manejo de circunstancias reales significativas para el que se educa, que lo llevan a desempeñar exitosamente las labores especificas en los roles asumidos.

Es decir, que el ser humano aprende a hacer, pero además también tiene la capacidad de aprender a aprender, pues las literacidades reúnen un conjunto de conocimientos, saberes e intereses que llevarán al sujeto en definitiva a saber actuar dentro de su ejercicio profesional. 
El capital humano juega un papel relevante en el progreso económico, pero se desestima la importancia de que además, contribuya en el incremento y mejoramiento de la calidad de vida al generar mayor bienestar social en la población impactada, pues la formación actual debe contribuir no solo a formar un buen trabajador, sino además a preparar personas que mejoren sus relaciones privadas, sociales, públicas, políticas y ciudadanas. Desde esta perspectiva el emprendimiento social contribuye a desarrollar con éxito las propuestas de transformación social resaltando los valores y las prácticas sociales, mediante la puesta en marcha de proyectos innovadores que generen crecimiento económico de manera sostenible, justa y respetuosa.

Eso indica dos situaciones: la primera, que el saber se construye y avanza a través de la interacción social, por lo cual las personas son susceptibles de educarse y desarrollarse bajo modelos específicos de formación y, por otro lado, que no dependen de una apreciación individual, sino que se encuentran en un ambiente social amplio que permite analizarlas a través de prácticas observables mientras se toman decisiones sobre situaciones concretas susceptibles de ser mejoradas.

Es por este motivo que no se persigue con esta propuesta subordinar la educación a una demanda de mercado laboral, por el contrario, se cree que esta propuesta debe colaborar para brindar la orientación personal, social y económica que ayude a los interesados a vivir plenamente desde el reconocimiento y desarrollo de sus capacidades, que puedan conocer y defender sus derechos para continuar siendo autónomos valorando los avances y saberes de los otros.

Desde la teoría motivacional, el emprendedor social es una persona que demuestra una gran necesidad de alcanzar su realización personal. De esta surge la teoría de la escuela comporta mentalista (McClelland, David, 2000) que arroja como principales características presentes en un empresario exitoso (y que luego han sido adoptadas por las diferentes teorías que quieren describir la personalidad de un emprendedor) las siguientes:

1) Búsqueda de oportunidad e iniciativa.

2) Persistencia.

3) Correr riesgos calculados

4) Exigencia de calidad y eficiencia

5) Involucrarse.

6) Búsqueda de informaciones. 
7) Establecimiento de metas.

8) Planeamiento y monitoreo sistemáticos.

9) Persuasión y red de contactos.

10) Independencia y auto confianza.

Estas características se presumen que son desarrolladas desde una formación compleja que alienta el desarrollo de habilidades en emprendimiento social toda vez que se entiende que un emprendedor social, según (Kliksberg, 2011) es una persona que demuestra una gran necesidad de alcanzar su realización personal. Un emprendedor social es un individuo que no pierde sus ilusiones, una persona cargada de energía y de creatividad, orientada completamente a lograr la meta propuesta, cree en sí mismo y confía en sus capacidades (Kliksberg, 2011). Es una persona que se compromete a desarrollar una tarea totalmente, no le teme a enfrentar riesgos moderados, es autónomo y ama su independencia en la toma de decisiones.

El modelo de formación en emprendimiento social estimula la toma de conciencia de los estudiantes acerca de que el conocimiento no está ajeno a su desarrollo en la vida, ni de los demás y que lo que da sentido a su ser es "el contexto y el objeto, es decir, vivir, pensar y sentir la realidad” (Morin, Edgar, 2011).

El llegar a perfeccionar las habilidades que como joven ayudan a alcanzar una formación integral, implica al docente formar en la conciencia que si el conocimiento es descontextualizado no es parte de una formación útil, asertiva, actual, productiva y comprometida. (Cadena, 2017).

Parafraseando a (Morín 2011) se afirma que reformar la educación implica enseñar a integrar el conocimiento en la cotidianidad de la vida. 


\section{REFERENCIAS BIBLIOGRÁFICAS}

Bauman, Z. (2007). Miedo liquido. La sociedad contemporánea y sus temores. En Z. Bauman, Miedo liquido. La sociedad contemporánea y sus temores. (pág. 231). Barcelona: Paidòs.

Bauman, Zygmunt. (2007). Miedo Liquido. La sociedad contemporánea y sus temores. Barcelona.: Paidòs.

Cadena, L. F. (2017). Desde la gestión educativa y la articulación de la educación técnica, un modelo de emprendimiento social para la educación media técnica. Tesis doctoral. Bogota.

Dees, G. E. (2001). Enterprising Nonprofits a toolkit for social entrepreneurs. New York: Jhon Wiley and Sons Inc.

DNP, D. N. (20 de noviembre de 2016). Ramking de las ciudades con mayor desempleo juvenil. Obtenido de https://www.dnp.gov.co/Paginas/Ranking-de-las-ciudades-con-mayor-desempleojuvenil.aspx

DNP, D. N. (20 de noviembre de 2016). Ranking de las ciudades con mayor desempleo juvenil. Obtenido de https:/www.dnp.gov.co/Paginas/Ranking-de-las-ciudades-con-mayor-desempleojuvenil.asp

Gobierno de Colombia. (10 de 06 de 2018). Obtenido de Colombia joven: http://www.colombiajoven.gov.co/Paginas/default.aspx

Gonfiantini, V. (16 de 10 de 2016). multiversidadreal.edu.mx. Obtenido de http://www.multiversidadreal.edu.mx/wp-content/uploads/2016/05/Tesis-GonfiantiniVirginia.pdf

Kliksberg, B. (2011). Emprendedores sociales. Los que hacen la diferencia. En B. Kliksberg. Buenos Aires.: Fundación CLARITAS.

McClelland, D. (2.000). Gestión de desempeño basado en competencias. Washington.: Organización Panamericana de la Salud.

McClelland, David. (2000). Gestión del desempeño basado en competencias. Washington.: Organización Panamericana de la Salud.

MEN, M. d. (27 de 11 de 2016). Plan Decenal de Educación 2006-2016. Obtenido de PNDE final.: http://www.plandecenal.edu.co/cms/media/herramientas/PNDE\%20FINAL_ISBN\%20we b.pdf

MEN, M.d. (01 de 12 de 2016). Todos por un nuevo país. Obtenido de http://www.mineducacion.gov.co/1759/w3-propertyvalue-56819.html

MEN, M. d. (16 de octubre de 2017). Lineamientos de articulación de la educación media técnica. Obtenido de https://www.mineducacion.gov.co/1621/articles-299165_archivo_pdf_Lineamientos.pdf

MEN, S. (S.F.). Ministerio de Educación Nacional. Recuperado el 17 de FEBRERO de 2017, de http://www.mineducacion.gov.co/sistemasdeinformacion

Morin, E. (2011). Como vivir en tiempos de crisis. En E. Morin. Buenos Aires: Ediciones Nueva Edición. 
Luisa Fernanda Cadena-Corredor

Morin, E. (2011). La Via. Barcelona. : Paidòs.

Morin, E. (2015). Enseñar a vivir. Buenos Aires.: Nueva Visión.

Morin, Edgar. (2011). Como vivir en tiempos de crisis. Buenos Aires: Ediciones Nueva Visión.

Morin, Edgar. (2014). Enseñar a vivir. Manifiesto para cambiar la educación. En E. Morin. Buenos Aires: Ediciones Nueva Visión.

Morin, Edgar. (2015). Enseñar a vivir. Buenos Aires: Ediciones Nueva Visión. 